Kragujevac Journal of Mathematics

Volume 42(2) (2018), Pages 177-191.

\title{
TERNARY HYPERGROUPS OF TYPE U ON THE RIGHT
}

\author{
B. DAVVAZ ${ }^{1}$, F. DEHGHAN ${ }^{1}$, AND M. FARSHI $^{1}$
}

\begin{abstract}
In this paper, it will be generalized the notion of hypergroups of type $\mathrm{U}$ on the right to ternary hypergroups of type $\mathrm{U}$ on the right and some of their properties will be investigated. We will determine all ternary hypergroups of size 2 and 3. Quotient ternary hypergroups of type $\mathrm{U}$ on the right, by defining a regular relation, are considered and it will be proved that arisen ternary hypergroup is right reversible.
\end{abstract}

\section{INTRODUCTION AND BASIC DEFINITIONS}

In this section, after gathering the history and related works, we review all definitions and simple properties we require of hyperstructures. The hyperstructure theory was emerged in 1934, when Marty introduced the notion of a hypergroup [20]. Later on this subject studied by many mathematicians. Hyperstructure theory both extends some well-known group results and leads us to a wide variety of applications. The principal notions of the theory of hyperstructures can be found in [3-5,23]. As we know, in a classical group, the composition of two elements is an element, while in a hypergroup, the composition of two elements is a set, i.e., in a hyperstructure $(H, *)$ we have $x * y \subseteq H$, for each two elements $x, y \in H$.

$n$-ary generalizations of algebraic structures is the most natural way to study their fundamental properties. It seems that early research on $n$-ary algebras goes back to Krasner's lecture at the 53rd annual meeting of the American Association of the Advancement of Science in 1904. Recently, a research about $n$-hypergroups as a suitable generalization of hypergroups in the sense of Marty has been inchoated by Davvaz and Vougiouklis [7]. Then, this concept has been studied by many authors,

Key words and phrases. Hypergroup of type $\mathrm{U}$ on the right, Ternary hypergroup of type $\mathrm{U}$ on the right, Regular hypergroup, Reversible hypergroup.

2010 Mathematics Subject Classification. Primary: 05A99. Secondary: 20 N20.

Received: January 23, 2016.

Accepted: January 19, 2017. 
for example see Anvariyeh et al. [1,2], Ghadiri and Waphare [24], Leoreanu-Fotea and Davvaz [6,17-19], Mirvakili and Davvaz [21,22], Davvaz et al. [8,9].

In [12], the concept of hypergroups of type $U$ on the right has been introduced in order to analyze properties of quotient hypergroups $H / h$ of a hypergroup $H$ with respect to a subhypergroup $h \subseteq H$ ultraclosed on the right. In [13], Freni showed that if $H$ is a finite hypergroup of type $\mathrm{U}$ on the right such that its right scalar identity is also a left identity, then all sub-semihypergroups of $H$ are also hypergroups. In [14], it has been proved that every right scalar identity of a hypergroup of type $U$ on the right of size $\leq 5$ is also a left identity. Also, some examples of hypergroups of type $\mathrm{U}$ on the right of size $\geq 6$ where a right scalar identity is not a left identity has been presented. A characterization of hypergroups of type $U$ on the right of size 5 can be found in [15,16]. Also see [11].

Let $H$ be a non-empty set, $n \geq 2$ a natural number, and $\mathcal{P}^{*}(H)$ be the set of all non-empty subsets of $H$. An $n$-hyperoperation on $H$ is a mapping $f: H^{n} \rightarrow \mathcal{P}^{*}(H)$, where $H^{n}$ is the $n$-times cartesian product of $H$. If $A_{1}, \ldots, A_{n}$ are non-empty subsets of $H$, then we define

$$
f\left(A_{1}, \ldots, A_{n}\right)=\bigcup_{x_{i} \in A_{i}} f\left(x_{1}, \ldots, x_{n}\right) .
$$

We shall use the notation $x_{i}^{j}$ to denote the sequence $x_{i}, x_{i+1}, \ldots, x_{j}$.

Let $H$ be a non-empty set and $f: H^{n} \rightarrow \mathcal{P}^{*}(H)$ be an $n$-hyperoperation.

(1) The couple $(H, f)$ is called an n-hypergroupoid.

(2) An $n$-hypergroupoid $(H, f)$ is called an $n$-semihypergroup if the following associative axiom holds:

$$
f\left(x_{1}^{i-1}, f\left(x_{i}^{n+i-1}\right), x_{n+i}^{2 n-1}\right)=f\left(x_{1}^{j-1}, f\left(x_{j}^{n+j-1}\right), x_{n+j}^{2 n-1}\right),
$$

for every $i, j \in\{1, \ldots, n\}$ and for every $x_{1}^{2 n-1} \in H$.

(3) An $n$-semihypergroup $(H, f)$ is called an n-hypergroup if $f\left(x_{1}^{i-1}, H, x_{i+1}^{n}\right)=H$, for every $i \in\{1, \ldots, n\}$ and $x_{1}^{n} \in H$. The above condition is called the reproduction axiom.

An $n$-hypergroup $(H, f)$ is called a hypergroup if $n=2$ and it is called a ternary hypergroup if $n=3$.

Whenever a semihypergroup $(H, \circ)$ contains an element $e$ with the property that, for all $x \in H$, one has $x \in x \circ e$ (resp. $x \in e \circ x$ ), then we say that $e$ is a right identity (resp. left identity) of $H$. The element $e$ is called an identity, if $x \in x \circ e \cap e \circ x$ holds, for all $x \in H$. If $x \circ e=\{x\}$ (resp. $e \circ x=\{x\}$ ), for all $x \in H$, then $e$ is called a right scalar identity (resp. left scalar identity).

Definition 1.1. [15] A hypergroup $(H, o)$ is said to be of type $\mathrm{U}$ on the right if it fulfills the following conditions:

(1) $H$ has a right scalar identity $e$,

(2) $x \in x \circ y$ implies that $y=e$, for all $x, y \in H$. 
Example 1.1. [10] We know that every group can be considered as a hypergroup. It is not difficult to see that the groups $\left(\mathbb{Z}_{2},+_{2}\right)$ and $\left(\mathbb{Z}_{3},+_{3}\right)$ are hypergroups of type $\mathrm{U}$ on the right.

Example 1.2. [10] Let $\mathbb{S}_{3} / \mathbb{S}_{2}$ be the set of all cosets of the subgroup $\mathbb{S}_{2}=\left\langle\left(\begin{array}{ll}1 & 2\end{array}\right)\right\rangle$ of the symmetric group $\mathbb{S}_{3}$, i.e., $\mathbb{S}_{3} / \mathbb{S}_{2}=\left\{\mathbb{S}_{2},\left(\begin{array}{ll}1 & 3\end{array}\right) \mathbb{S}_{2},\left(\begin{array}{ll}2 & 3\end{array}\right) \mathbb{S}_{2}\right\}$. Set $e=\mathbb{S}_{2}, x=\left(\begin{array}{ll}1 & 3\end{array}\right) \mathbb{S}_{2}$, and $y=(23) \mathbb{S}_{2}$. Then, $\mathbb{S}_{3} / \mathbb{S}_{2}$ with the following table is a hypergroup of type $\mathrm{U}$ on the right.

\begin{tabular}{c|ccc}
$*$ & $e$ & $x$ & $y$ \\
\hline$e$ & $\{e\}$ & $\{x, y\}$ & $\{x, y\}$ \\
$x$ & $\{x\}$ & $\{e, y\}$ & $\{e, y\}$ \\
$y$ & $\{y\}$ & $\{e, x\}$ & $\{e, x\}$
\end{tabular}

\section{TERNARY HYPERGROUPS OF TYPE U ON THE RIGHT}

In this section, we introduce the notion of ternary hypergroups of type $U$ on the right which is a generalization of the idea presented by Freni et al. In this regards, several examples and properties of them will be expressed.

If a ternary hypergroup $(H, f)$ contains an element $e$ with the property that, for all $x \in H$, one has $x \in f(x, e, e)$ (resp. $x \in f(e, x, e)$ or $x \in f(e, e, x)$ ), then we say that $e$ is a right identity (resp. central identity or left identity) of $H$. The element $e$ is called an identity, if $x \in f(x, e, e) \cap f(e, x, e) \cap f(e, e, x)$. If for all $x \in H$, one has $f(x, e, e)=\{x\}$ (resp. $f(e, x, e)=\{x\}$ or $f(e, e, x)=\{x\})$, then $e$ is called a right scalar identity (resp. central scalar identity or left scalar identity).

Definition 2.1. A ternary hypergroup $(H, f)$ is called of type $\mathrm{U}$ on the right if it fulfills the following conditions:

$\left(U_{1}\right) H$ has a right scalar identity $e$,

$\left(U_{2}\right)$ for all $x, y \in H$, from $x \in f(x, e, y)$ it follows that $y=e$.

We shall use the notation $(H, f, e)$ to say that $e$ is a right scalar identity.

Example 2.1. Let $(H, \circ)$ be a hypergroup of type $U$ on the right. We consider a ternary hyperoperation $f$ on $H$ as follows:

$$
f(x, y, z)=x \circ y \circ z, \quad \text { for all } x, y, z \in H .
$$

Then, $(H, f)$ is a ternary hypergroup of type $\mathrm{U}$ on the right. $(H, f)$ is called the ternary hypergroup on the right extracted from $(H, \circ)$ and shall be denoted by $\operatorname{ext}(H)$.

In the next theorem we will show a special construction of a hypergroup of type $U$ on the right starting from a ternary hypergroup of type $U$ on the right. We premise a proposition that has already been demonstrated in [8].

Proposition 2.1. An n-semihypergroup $(H, f)$ is an n-hypergroup if and only if for some $a \in H$ and all $b, c \in H$ there exist $x, y \in H$ such that

$$
b \in f(c, \stackrel{(n-2)}{a}, x) \cap f(y, \stackrel{(n-2)}{a}, c) .
$$


Proof. See Proposition 2.2 in [8].

Theorem 2.1. Let $(H, f)$ be a ternary semihypergroup. Then, the following assertions are equivalent:

(1) $(H, f, e)$ is a ternary hypergroup of type $U$ on the right,

(2) $(H, \circ, e)$ is a hypergroup of type $U$ on the right, where

$$
x \circ y=f(x, e, y), \quad \text { for all } x, y \in H .
$$

$(H, \circ, e)$ is called the hypergroup induced by $(H, f, e)$.

Proof. (1) $\Rightarrow(2)$ Since $f$ is associative, it ensues that $\circ$ is associative. For each $x \in H$ we have $x \circ H=f(x, e, H)=H$ and $H \circ x=f(H, e, x)=H$, whence $(H, \circ)$ is a hypergroup. Moreover, for each $x \in H$ we have $x \circ e=f(x, e, e)=\{x\}$. Now, let $x \in x \circ y$. Then, $x \in f(x, e, y)$, which implies that $y=e$.

$(2) \Rightarrow(1)$ Let $b, c \in H$ be arbitrary elements. Set $a=e$. Since $(H, \circ)$ is a hypergroup, there exists $x \in H$ such that $b \in c \circ x=f(c, e, x)$. Also, there exists $y \in H$ such that $b \in y \circ c=f(y, e, c)$. So, $b \in f(c, e, x) \cap f(y, e, c)$ and therefore by Proposition 2.1, $(H, f)$ is a ternary hypergroup. For all $x \in H$ we have $f(x, e, e)=x \circ e=\{x\}$. Let $x \in f(x, e, y)$, for some elements $x, y \in H$. Then, we have $x \in x \circ y$. Since $(H, \circ)$ is a hypergroup of type $\mathrm{U}$ on the right, we deduce that $y=e$. This completes the proof.

We recall that the ternary hypergroup extracted from a hypergroup $(H, \circ)$ is $(H, f)$, where $f(x, y, z)=x \circ y \circ z$, for all $x, y, z \in H$. Note that the hypergroup induced by $\left(\mathbb{S}_{3} \backslash \mathbb{A}_{3}, f\right)$, defined in Example 2.4, is equal to $\left(\mathbb{Z}_{3},+_{3}\right)$. But, the ternary hypergroup extracted from $\left(\mathbb{Z}_{3},+_{3}\right)$ is not equal to $\left(\mathbb{S}_{3} \backslash \mathbb{A}_{3}, f\right)$.

Example 2.2. Let $G$ be a group and let $H$ be a subgroup of $G$. Then, the quotient set $G / H$ is a ternary hypergroup of type $\mathrm{U}$ on the right with respect to the ternary hyperoperation $f$ defined by

$$
f(x H, y H, z H)=\{t H \mid t \in x H y H z\} .
$$

It is easy to see that $H$ is the identity element of $(H, f)$.

Example 2.3. Let $f$ be a ternary hyperoperation on $\mathbb{Z}$ as follows:

$$
f(x, y, z)=\{x+y+z, x+y-z, x-y+z, x-y-z\} .
$$

It is easy to check that $(\mathbb{Z}, f)$ is a ternary hypergroup of type $U$ on the right. The element 0 is a right identity but it is not a left and central identity.

Example 2.4. Let $\mathbb{S}_{3} \backslash \mathbb{A}_{3}=\left\{\left(\begin{array}{l}1 \\ 2\end{array}\right),\left(\begin{array}{ll}1 & 3\end{array}\right),\left(\begin{array}{l}2 \\ 2\end{array}\right)\right\}$ be the set of all odd permutations of $\mathbb{S}_{3}$. Set $e=\left(\begin{array}{ll}1 & 2\end{array}\right), x=\left(\begin{array}{ll}1 & 3\end{array}\right)$ and $y=\left(\begin{array}{ll}2 & 3\end{array}\right)$. Then, $\left(\mathbb{S}_{3} \backslash \mathbb{A}_{3}, f\right)$ is a ternary group of 
type $\mathrm{U}$ on the right, where $f$ is defined as follows:

$$
\begin{aligned}
f(e, e, e) & =f(e, x, x)=f(x, e, y)=f(e, y, y)=f(x, x, e)=f(y, e, x)=f(y, y, e) \\
=f(x, y, x) & =f(y, x, y)=\{e\} \\
f(e, x, y) & =f(y, x, e)=f(x, x, x)=f(e, e, x)=f(x, e, e)=f(y, e, y)=f(e, y, e) \\
=f(x, y, y) & =f(y, y, x)=\{x\} \\
f(e, y, x) & =f(y, y, y)=f(e, e, y)=f(y, e, e)=f(x, e, x)=f(x, y, e)=f(e, x, e) \\
& =f(x, x, y)=f(y, x, x)=\{y\} .
\end{aligned}
$$

\section{Ternary hypergroups of type U on the Right of SIZE 2 AND 3}

In this section, we determine all ternary hypergroups of type $U$ on the right of size 2 and 3.

Definition 3.1. Let $(H, f, e)$ be a ternary hypergroup of type $\mathrm{U}$ on the right. Then, an element $x \in H$ is said to be a $T$-element if

$$
f(x, e, y)=H \backslash\{x\}, \quad \text { for all } y \in H \backslash\{e\} .
$$

Example 3.1. Let $f$ be a ternary hyperoperation defined on $H=\{e, x, y\}$ as follows:

$$
\begin{aligned}
& f(x, e, x)=f(x, e, y)=f(x, y, e)=f(x, x, e)=\{e, y\}, \\
& f(y, e, x)=f(y, e, y)=f(y, x, e)=f(y, y, e)=\{e, x\}, \\
& f(e, e, x)=f(e, e, y)=f(e, x, e)=f(e, y, e)=\{x, y\}, \\
& f(x, y, y)=f(x, y, x)=f(x, x, x)=f(x, x, y)=f(e, x, x)=f(e, x, y)=H, \\
& f(e, y, x)=f(e, y, y)=f(y, x, x)=f(y, x, y)=f(y, y, x)=f(y, y, y)=H, \\
& f(x, e, e)=x, f(y, e, e)=y, f(e, e, e)=e .
\end{aligned}
$$

It is easy to check that $(H, f)$ is a ternary hypergroup of type $\mathrm{U}$ on the right and each element of $H$ is a $T$-element.

Proposition 3.1. Let $(H, f, e)$ be a ternary hypergroup of type $U$ on the right with at least two elements. Let $x \in H$ be a T-element. Then, the following assertions hold.

(1) For all $y, z \in H \backslash\{e\}$ we have $e \in f(y, e, z)$.

(2) If $|H| \geq 3$, then for all $y, z \in H \backslash\{e\}$ we have $|f(y, e, z)| \geq 2$.

Proof. (1) It is a direct consequence of Lemma 3.7 (3).

(2) Let $y, z \in H \backslash\{e\}$ be arbitrary elements. By reproduction axiom, there exists $w \in H$ such that $x \in f(w, e, y)$. Since $(H, f)$ is a ternary hypergroup of type $\mathrm{U}$ on the right, we have $w \neq x$. Thus, as $x$ is a $T$-element, we have

$$
H \backslash\{x\}=f(x, e, z) \subseteq f(f(w, e, y), e, z)=f(w, e, f(y, e, z)) .
$$

If $|f(y, e, z)|=1$, then by assertion (1) we have $f(y, e, z)=e$ and therefore $H \backslash\{x\}=w$. This implies that $H=\{x, w\}$, a contradiction.

Theorem 3.1. Let $(H, f, e)$ be a ternary hypergroup of type $U$ on the right with $|H|=2$. Then, $H=\operatorname{ext}\left(\mathbb{Z}_{2}\right)$. (See Example 1.1 and 2.1.) 
Proof. Let $H=\{e, x\}$. By condition $\left(U_{1}\right)$ of Definition 2.1 we have $f(x, e, e)=\{x\}$ and $f(e, e, e)=\{e\}$. By using Lemma 3.1, we have

$$
f(e, x, e)=f(e, e, x)=\{x\}, \quad f(x, e, x)=f(x, x, e)=\{e\} .
$$

We claim that $f(e, x, x)=\{e\}$. If not, then we have $x \in f(e, x, x)$ which implies that

$$
\{x\}=f(x, e, e) \subseteq f(f(e, x, x), e, e)=f(e, f(x, x, e), e)=f(e, e, e)=\{e\},
$$

a contradiction. Also, we claim that $f(x, x, x)=\{x\}$. If not, then we have $e \in$ $f(x, x, x)$ and so

$$
\{e\}=f(e, e, e) \subseteq f(e, f(x, x, x), e)=f(e, x, f(x, x, e))=f(e, x, e)=\{x\},
$$

a contradiction. Therefore, the desired result holds.

Theorem 3.2. Let $(H, f, e)$ be a ternary hypergroup of type $U$ on the right with $|H|=3$. Then, either $H=\operatorname{ext}\left(\mathbb{Z}_{3}\right)$ or $H=\operatorname{ext}\left(\mathbb{S}_{3} / \mathbb{S}_{2}\right)$ or $H=\operatorname{ext}\left(\mathbb{S}_{3} \backslash \mathbb{A}_{3}\right)$. (See Example 1.1, 1.2, 2.1 and 2.4.)

Proof. Let $H=\{e, x, y\}$. It is obvious that $f(e, e, x) \neq H$ and $f(e, e, y) \neq H$. We claim that $|f(e, e, x)|=|f(e, e, y)|$. If this is not the case, then without loss of generality we can assume that $|f(e, e, x)|=1$ and $|f(e, e, y)|=2$. Thus, we have $f(e, e, y)=\{x, y\}$ and by Lemma 3.2 we have $f(e, e, x)=x$. By reproduction axiom, there exists $z \in H$ such that $y \in f(x, e, z)$. Hence,

$$
x \in f(e, e, y) \subseteq f(e, e, f(x, e, z))=f(f(e, e, x), e, z)=f(x, e, z),
$$

which implies that $z=e$. From $y \in f(x, e, z)$ it follows that $x=y$, a contradiction. Thus, we have the following two cases.

Case 1. Let $f(e, e, x)=f(e, e, y)=\{x, y\}$. Then, we have

$$
\begin{aligned}
f(x, e, y) & =f(x, f(e, e, e), y)=f(x, e, f(e, e, y))=f(x, e, f(e, e, x)) \\
& =f(x, f(e, e, e), x)=f(x, e, x) .
\end{aligned}
$$

So, $f(x, e, y)=f(x, e, x)$. Since $x \notin f(x, e, y)$ we have $f(x, e, y) \subseteq\{e, y\}$. In the case that $f(x, e, y)=f(x, e, x)=\{e\}$, we have

$$
\{e, x\}=f(x, e, x) \cup f(x, e, y) \cup f(x, e, e)=f(x, e, H)=H,
$$

which is a contradiction. In the case that $f(x, e, y)=f(x, e, x)=\{y\}$, we have

$$
\{y\}=f(x, e, y)=f(x, e, f(x, e, y))=f(f(x, e, x), e, y)=f(y, e, y) .
$$

This implies that $y=e$ which is a contradiction. Thus, $f(x, e, y)=\{e, y\}$. In a similar manner we can prove that

$$
\begin{aligned}
& f(x, e, x)=f(x, e, y)=f(x, y, e)=f(x, x, e)=\{e, y\} \\
& f(y, e, x)=f(y, e, y)=f(y, x, e)=f(y, y, e)=\{e, x\}, \\
& f(e, e, x)=f(e, e, y)=f(e, x, e)=f(e, y, e)=\{x, y\} \\
& f(x, y, y)=f(x, y, x)=f(x, x, x)=f(x, x, y)=f(e, x, x)=f(e, x, y)=H, \\
& f(e, y, x)=f(e, y, y)=f(y, x, x)=f(y, x, y)=f(y, y, x)=f(y, y, y)=H .
\end{aligned}
$$


Since $(H, f)$ is a ternary hypergroup of type $\mathrm{U}$ on the right, we have

$$
f(x, e, e)=\{x\}, \quad f(y, e, e)=\{y\}, \quad f(e, e, e)=\{e\} .
$$

In this case we have $H=\operatorname{ext}\left(\mathbb{S}_{3} / \mathbb{S}_{2}\right)$.

Case 2. Let $|f(e, e, x)|=f(e, e, y) \mid=1$. By Lemma 3.2, we have $f(e, e, x)=\{x\}$ and $f(e, e, y)=\{y\}$. By Lemma 3.8, we have $|f(e, x, e)|=|f(e, y, e)|=1$. By reproduction axiom we have

$$
f(e, e, e) \cup f(e, x, e) \cup f(e, y, e)=H .
$$

So, there exist the following two subcases.

Subcase 2.1. Suppose that $f(e, x, e)=\{x\}$ and $f(e, y, e)=\{y\}$. Since $x \notin f(x, e, x)$ we have $f(x, e, x) \subseteq\{e, y\}$. We claim that $f(x, e, x)=\{y\}$. If $f(x, e, x)=\{e\}$, then as $f(e, e, x\} \cup f(x, e, x) \cup f(y, e, x)=f(H, e, x)=H$, we have $y \in f(y, e, x)$ which implies that $x=e$, a contradiction. If $f(x, e, x)=\{e, y\}$, then from the following equalities we conclude that $y \notin f(x, e, y)$ :

$$
\begin{aligned}
\{x\} \cup f(y, e, x) & =f(e, e, x) \cup f(y, e, x)=f(\{e, y\}, e, x)=f(f(x, e, x), e, x) \\
& =f(x, e, f(x, e, x))=f(x, e, e) \cup f(x, e, y),
\end{aligned}
$$

On the other hand, by condition $\left(U_{2}\right), x \notin f(x, e, y)$. Thus, $f(x, e, y)=\{e\}$. Therefore,

$$
\begin{aligned}
y \in f(e, e, y) \cup f(y, e, y) & =f(\{e, y\}, e, y)=f(f(x, e, x), e, y) \\
& =f(x, e, f(x, e, y))=\{x\},
\end{aligned}
$$

that it is a contradiction. Hence, $f(x, e, x)=\{y\}$. In a similar manner we have that $f(y, e, y)=\{x\}$. By an argument as above, we have

$$
\begin{aligned}
f(y, e, y) & =f(e, y, y)=f(y, y, e)=f(x, y, x)=f(x, x, y)=f(y, x, x)=\{x\}, \\
f(x, x, x) & =f(e, y, x)=f(x, y, e)=f(e, x, y)=f(y, e, x)=f(y, y, y) \\
& =f(y, x, e)=f(x, e, y)=\{e\}, \\
f(x, x, e) & =f(x, e, x)=f(x, y, y)=f(y, x, y)=f(y, y, x)=f(e, x, x)=\{y\} .
\end{aligned}
$$

So, in this case we have $H=\operatorname{ext}\left(\mathbb{Z}_{3}\right)$.

Subcase 2.2. Suppose that $f(e, x, e)=\{y\}$ and $f(e, y, e)=\{x\}$. By an argument similar to that in subcase 2.1, we have $f(x, e, x)=\{y\}$ and $f(y, e, y)=\{x\}$. Moreover, we have

$$
\begin{aligned}
f(e, x, y) & =f(y, x, e)=f(x, y, y)=f(x, x, x)=f(y, y, x)=\{x\}, \\
f(e, y, x) & =f(y, x, x)=f(x, y, e)=f(x, x, y)=f(y, y, y)=\{y\}, \\
f(y, e, x) & =f(x, e, y)=f(y, e, x)=f(y, y, e)=f(x, x, e)=f(x, y, x) \\
& =f(y, x, y)=f(e, x, x)=\{e\} .
\end{aligned}
$$

So, in this case we have $H=\operatorname{ext}\left(\mathbb{S}_{3} \backslash \mathbb{A}_{3}\right)$. 
Lemma 3.1. Let $(H, f)$ be a ternary hypergroup of type $U$ on the right. Then, for every $x, y \in H$ from $x \in f(x, y, e)$ it follows that $y=e$.

Proof. Let $x, y$ be arbitrary elements of $H$. Then, we have

$$
x \in f(x, y, e)=f(f(x, e, e), y, e)=f(x, e, f(e, y, e)) .
$$

By reproduction axiom, there exists $z \in f(e, y, e)$ such that $x \in f(x, e, z)$. By condition $\left(U_{2}\right)$ of Definition 2.1 we have $z=e$. Hence,

$$
e \in f(e, e, e) \subseteq f(e, f(e, y, e), e)=f(e, e, y) .
$$

Again, condition $\left(U_{2}\right)$ implies that $y=e$.

Lemma 3.2. Let $(H, f)$ be a ternary hypergroup of type $U$ on the right and let $x \in H$. Then, the following assertions are equivalent:

(1) $|f(e, e, x)|=1$,

(2) $f(e, e, x)=\{x\}$.

Proof. (1) $\Rightarrow(2)$ Let $f(e, e, x)=\{y\}$. Then, we have

$$
f(e, e, y)=f(e, e, f(e, e, x))=f(f(e, e, e), e, x)=f(e, e, x)=\{y\} .
$$

By reproduction axiom, there exists $z \in H$ such that $y \in f(x, e, z)$. Thus,

$$
\{y\}=f(e, e, y) \subseteq f(e, e, f(x, e, z))=f(f(e, e, x), e, z)=f(y, e, z) .
$$

This follows that $z=e$. Hence $y=x$.

$(2) \Rightarrow(1)$ It is trivial.

Lemma 3.3. Let $(H, f, e)$ be a ternary hypergroup of type $U$ on the right. Then, for each $x \in H \backslash\{e\}$ the following assertions are equivalent:

(1) $x \notin f(e, e, H \backslash\{x\})$,

(2) $f(e, e, x)=\{x\}$.

Proof. $(1) \Rightarrow(2)$ Let $x \notin f(e, e, H \backslash\{x\})$. By reproduction axiom, we have $f(e, e, H)=$ $H$. This implies that $x \in f(e, e, x)$. Let $y \in f(e, e, x)$ be an arbitrary element. We have to show that $y=x$. By reproduction axiom, there exists $z \in H$ such that $x \in f(y, e, z)$. Whence,

$$
x \in f(y, e, z) \subseteq f(f(e, e, x), e, z)=f(e, e, f(x, e, z)) .
$$

So, there exists $t \in f(x, e, z)$ such that $x \in f(e, e, t)$. Since $x \notin f(e, e, H \backslash\{x\})$, we obtain $x=t$. Thus, $x \in f(x, e, z)$ and by condition $\left(U_{2}\right)$ we have $z=e$. Now, since $x \in f(y, e, z)$ we have $y=x$.

$(2) \Rightarrow(1)$ By way of contradiction, we suppose that there exists $z \in H \backslash\{x\}$ such that $x \in f(e, e, z)$. By reproduction axiom, there exists $y \in H \backslash\{e\}$ such that $z \in f(x, e, y)$ and so,

$$
f(e, e, z) \subseteq f(e, e, f(x, e, y))=f(f(e, e, x), e, y)=f(x, e, y) .
$$

Whence, $x \in f(x, e, y)$. Now, from condition $\left(U_{2}\right)$ it follows that $y=e$ and from $z \in f(x, e, y)$ it follows that $z=x$, which is a contradiction. 
Lemma 3.4. Let $(H, f, e)$ be a ternary hypergroup of type $U$ on the right and let $e$ be a central identity. Then,

(1) $f(e, e, x)=f(e, x, e)$, for all $x \in H$,

(2) $f(x, e, y)=f(x, y, e)$, for all $x, y \in H$.

Proof. (1) Let $x$ be an arbitrary element of $H$. Then, we have

$$
f(e, x, e) \subseteq f(e, f(e, x, e), e)=f(e, e, f(x, e, e))=f(e, e, x) .
$$

Now, we show the reverse inclusion, i.e.,

$$
f(e, e, x) \subseteq f(e, e, f(e, x, e))=f(f(e, e, e), x, e)=f(e, x, e) .
$$

This completes the proof.

(2) For each $x, y \in H$ we have

$$
f(x, y, e) \subseteq f(x, f(e, y, e), e)=f(x, e, f(y, e, e))=f(x, e, y) .
$$

On the other hand, we have

$$
f(x, e, y) \subseteq f(x, e, f(e, y, e))=f(f(x, e, e), y, e)=f(x, y, e) .
$$

This completes the proof.

Lemma 3.5. Let $(H, f, e)$ be a ternary hypergroup of type $U$ on the right. Then, for each $x \in H \backslash\{x\}$ the following assertions are equivalent:

(1) $x \notin f(e, H \backslash\{x\}, e)$,

(2) $f(e, x, e)=\{x\}$.

Proof. By using Lemma 3.4, the proof follows similarly as the proof of Lemma 3.3.

The next lemma can be proved easily using previously defined notions and thus we omit its proof.

Lemma 3.6. Let $(H, f, e)$ be a ternary hypergroup of type $U$ on the right. Then, for every $x, y, z, t \in H$ the following assertions hold.

(1) $x \in f(e, e, y)$ implies that $f(t, z, x) \subseteq f(t, z, y)$.

(2) $x \in f(e, y, e)$ implies that $f(t, x, e) \subseteq f(t, e, y)$.

(3) If $f(e, e, x)=\{x\}$ and $z \in f(x, y, t)$, then $f(e, e, z) \subseteq f(x, y, t)$.

(4) If $f(e, x, e)=\{x\}$ and $z \in f(x, e, t)$, then $f(e, z, e) \subseteq f(x, t, e)$.

(5) If $x \in f(y, e, t)$ and $y \in f(x, e, z)$, then $e \in f(z, e, t) \cap f(t, e, z)$.

(6) If $e$ is a left identity and $x \in f(x, y, y)$, then $e \in f(e, y, y)$.

(7) If $e$ is a central identity and $x \in f(x, y, y)$, then $e \in f(y, e, y)$.

(8) If $f(e, e, x)=f(e, e, y)$, then $\left\{\begin{array}{l}f(z, t, x)=f(z, t, y), \\ f(z, x, t)=f(z, y, t), \\ f(t, x, z)=f(t, y, z), \\ f(t, z, x)=f(t, z, y) .\end{array}\right.$ 
(9) If $f(e, x, e)=f(e, y, e)$, then $\left\{\begin{array}{l}f(z, e, x)=f(z, e, y), \\ f(z, x, e)=f(z, y, e), \\ f(e, x, z)=f(e, y, z), \\ f(e, z, x)=f(e, z, y) .\end{array}\right.$

Lemma 3.7. Let $(H, f, e)$ be a ternary hypergroup of type $U$ on the right with at least two elements. Then, the following assertions hold.

(1) If $f(e, e, y)=H \backslash\{e\}$, then $f(x, e, y)=H \backslash\{x\}$, for every $x, y \in H$.

(2) If $f(e, y, e)=H \backslash\{e\}$, then $f(x, e, y)=f(x, y, e)=H \backslash\{x\}$, for every $x, y \in H$.

(3) If there exists $y \in H \backslash\{e\}$ such that $f(x, e, y)=H \backslash\{x\}$ for some $x \in H$, then $e \in f(y, e, z)$, for every $z \in H \backslash\{e\}$.

Proof. (1) Let $x, y \in H$ be arbitrary elements and $f(e, e, y)=H \backslash\{e\}$. Then, we have

$$
f(x, e, y)=f(x, f(e, e, e), y)=f(x, e, f(e, e, y))=f(x, e, H \backslash\{e\}) .
$$

This implies that $x \notin f(x, e, y)$. On the other hand, by using reproduction axiom we have

$$
\begin{aligned}
(H \backslash\{x\}) \cup\{x\}=H=f(x, e, H) & =f(x, e,(H \backslash\{e\}) \cup\{e\}) \\
& =f(x, e, H \backslash\{e\}) \cup f(x, e, e) \\
& =f(x, e, H \backslash\{e\}) \cup\{x\} \\
& =f(x, e, y) \cup\{x\} .
\end{aligned}
$$

This implies that $f(x, e, y)=H \backslash\{x\}$.

(2) The proof is similar to the proof of (1) and thus is omitted.

(3) Let $f(x, e, y)=H \backslash\{x\}$, where $x \in H$ and $y \in H \backslash\{e\}$. By way of contradiction, suppose that there exists $z \in H \backslash\{e\}$ such that $e \notin f(y, e, z)$. Then,

$$
x \notin f(x, e, f(y, e, z))=f(f(x, e, y), e, z)=f(H \backslash\{x\}, e, z) .
$$

By reproduction axiom, we have $H=f(H \backslash\{x\}, e, z) \cup f(x, e, z)$. This implies that $x \in f(x, e, z)$. So, by $\left(U_{2}\right)$ we have $z=e$, which is a contradiction.

Lemma 3.8. Let $(H, f, e)$ be a ternary hypergroup of type $U$ on the right. Assume further that $e$ is also a left scalar identity. Then, $|f(e, x, e)|=1$, for all $x \in H$.

Proof. By way of contradiction, suppose that $\{y, z\} \subseteq f(e, x, e)$ and $y \neq z$. Then,

$$
\{x\}=f(x, e, e)=f(f(e, e, x), e, e)=f(e, f(e, x, e), e)=f(e, y, e) \cup f(e, z, e) .
$$

Hence, $f(e, y, e)=f(e, z, e)=\{x\}$. On the other hand, we have

$$
\{y, z\}=f(e, x, e)=f(e, f(e, y, e), e)=f(e, e, f(y, e, e))=f(e, e, y)=\{y\},
$$

which is a contradiction. 


\section{QuOTIENT TERNARY HYPERGROUPS OF TYPE U ON THE RIGHT}

In the study of finite hypergroup $H$ of type $\mathrm{U}$ on the right the family $P_{\epsilon}=\{e x \mid x \in$ $H\}$, determined by the right scalar identity $\epsilon$ of $H$, plays a fundamental role (see $[15,16])$. In this section, we prove some results about the families $P_{e_{1}}=\{f(e, e, x) \mid x \in$ $H\}$ and $P_{e_{2}}=\{f(e, x, e) \mid x \in H\}$, where $(H, f, e)$ is a ternary hypergroup of type $\mathrm{U}$ on the right. We determine necessary and sufficient conditions such that $(H, f, e)$ is right reversible. Moreover, we define a regular relation $R$ on a ternary hypergroup such that the quotient set $H / R$ is a right reversible ternary hypergroup.

Definition 4.1. An element $x$ in a ternary hypergroup $(H, f)$ with right identity is said to be invertible if there exists an element $y \in H$ such that

$$
e \in f(x, e, y) \cap f(y, e, x) \cap f(x, y, e) .
$$

The set of all inverses of $x$ will be denoted by $x^{-1}$. A ternary hypergroup $(H, f)$ is called regular if it possesses an identity element and $x^{-1} \neq \emptyset$, for each $x \in H$. A regular ternary hypergroup $(H, f)$ is said to be right reversible if for every $x, y, z \in H$ with $x \in f(y, e, z)$, there exists $t \in z^{-1}$ such that $y \in f(x, e, t)$.

Theorem 4.1. Let $(H, f, e)$ be a ternary hypergroup of type $U$ on the right. Then, the following assertions are equivalent:

(1) e is a central identity,

(2) $(H, f)$ is right reversible.

Proof. (1) $\Rightarrow(2)$ By Lemma 3.4, $e$ is an identity element. Let $x$ be an arbitrary element of $H$. By reproduction axiom, there exists an element $y$ in $H$ such that $e \in f(x, e, y)$. We show that $y \in x^{-1}$. From

$$
x \in f(e, e, x) \subseteq f(f(x, e, y), e, x)=f(x, e, f(y, e, x))
$$

and

$$
x \in f(e, x, e) \subseteq f(f(x, e, y), x, e)=f(x, e, f(y, x, e)) .
$$

and the fact that $(H, f)$ is a ternary hypergroup of type $\mathrm{U}$ on the right, it follows that $f(y, e, x)=f(y, x, e)=e$. Thus, $(H, f)$ is regular. In order to show that $(H, f)$ is right reversible, let $x, y, z$ be arbitrary elements of $H$ and $x \in f(y, e, z)$. Then, by reproduction axiom, there exists $t \in H$ such that $y \in f(x, e, t)$. It suffices to show that $t \in z^{-1}$. By Lemma 3.6 (5), we have $e \in f(t, e, z) \cap f(z, e, t)$. On the other hand, we have

$$
t \in f(e, t, e) \subseteq f(f(t, e, z), t, e)=f(t, e, f(z, t, e)) .
$$

This implies that $f(z, t, e)=e$ which completes the proof.

$(2) \Rightarrow(1)$ It is trivial.

Let $R$ be a relation on a non-empty set $X$ and $A, B \subseteq X$. Then, $A \bar{R} B$ means that for every $a \in A$, there exists $b \in B$ such that $a R b$ and for every $b \in B$, there exists $a \in A$ such that $b R a$. 
An equivalence relation $R$ on a ternary hypergroupoid $(H, f)$ is called regular if

$$
x_{1} R y_{1}, x_{2} R y_{2}, x_{3} R y_{3} \Longrightarrow f\left(x_{1}, x_{2}, x_{3}\right) \bar{R} f\left(y_{1}, y_{2}, y_{3}\right),
$$

for all $x_{1}^{3}, y_{1}^{3} \in H$.

We describe ternary hypergroups of type $\mathrm{U}$ on the right with respect to $P_{e_{1}}=$ $\{f(e, e, x) \mid x \in H\}$ and $P_{e_{2}}=\{f(e, x, e) \mid x \in H\}$, where $e$ is a right identity.

Lemma 4.1. Let $(H, f, e)$ be a ternary hypergroup of type $U$ on the right such that $P_{e_{1}}$ is a partition of $H$. Then, $e$ is a left identity.

Proof. Let $x \in H$ be an arbitrary element. By reproduction axiom, there exists $y \in H$ such that $x \in f(e, e, y)$ and so we have

$$
f(e, e, x) \subseteq f(e, e, f(e, e, y))=f(e, f(e, e, e), y)=f(e, e, y) .
$$

Since $P_{e_{1}}$ is a partition of $H$, we deduce that $f(e, e, x)=f(e, e, y)$. Hence, $x \in$ $f(e, e, x)$.

Lemma 4.2. Let $(H, f, e)$ be a ternary hypergroup of type $U$ on the right such that $P_{e_{1}}$ is a partition of $H$. Then, $P_{e_{2}}$ is a partition of $H$.

Proof. It is obvious that $\bigcup_{x \in H} f(e, x, e)=H$. Suppose that $x \in f(e, y, e) \cap f(e, z, e)$, for $y, z \in H$. Then, we have

$$
\begin{aligned}
& f(e, x, e) \subseteq f(e, f(e, y, e), e)=f(e, e, f(y, e, e))=f(e, e, y), \\
& f(e, x, e) \subseteq f(e, f(e, z, e), e)=f(e, e, f(z, e, e))=f(e, e, z) .
\end{aligned}
$$

Since $P_{e_{1}}$ is a partition of $H$, we have $f(e, e, y)=f(e, e, z)$. Thus,

$$
\begin{aligned}
f(e, y, e) & =f(f(e, e, e), y, e)=f(e, f(e, e, y), e)=f(e, f(e, e, z), e)=f(f(e, e, e), z, e) \\
& =f(e, z, e) .
\end{aligned}
$$

This completes the proof.

Theorem 4.2. Let $(H, f, e)$ be a ternary hypergroup of type $U$ on the right such that $e$ is a central identity and $P_{e_{2}}$ is a partition of $H$. Then, the following assertions hold.

(1) The relation $R \subseteq H^{2}$ defined as follows is a regular relation

$$
x R y \Longleftrightarrow f(e, x, e)=f(e, y, e) .
$$

(2) The set $H / R$ endowed with the following ternary hyperoperation is a right reversible ternary hypergroup $\left.f\right|_{R}(R(x), R(y), R(z))=\{R(t) \mid t \in f(x, y, z)\}$, where $R(x)$ denotes the equivalence class of $x$.

(3) Let $R(y)=R(u), R(z)=R(v)$ and $R(t)=R(w)$, for some $y, z, t, u, v, w \in H$.

(a) The following statements are equivalent:

(i) $\left.R(x) \in f\right|_{R}(R(y), R(z), R(t))$,

(ii) $R(x) \cap f(u, v, w) \neq \emptyset$,

(iii) $R(x) \subseteq f(e, f(u, v, w), e)$. 
(b) If $|R(x)|=1$, for some $x \in H$, then the following statements are equivalent:

(i) $\left.R(x) \in f\right|_{R}(R(y), R(z), R(t))$,

(ii) $x \in f(u, v, w)$.

Proof. (1) Obviously, $R$ is an equivalence relation. Let $x_{1} R y_{1}, x_{2} R y_{2}$ and $x_{3} R y_{3}$. For each $x \in f\left(x_{1}, x_{2}, x_{3}\right)$, by using Lemma 3.4 , we have

$$
\begin{aligned}
x \in f(e, x, e) & \subseteq f\left(e, f\left(x_{1}, x_{2}, x_{3}\right), e\right) \subseteq f\left(e, f\left(x_{1}, f\left(e, x_{2}, e\right), x_{3}\right), e\right) \\
& =f\left(e, x_{1}, f\left(e, x_{2}, f\left(e, x_{3}, e\right)\right)\right)=f\left(e, x_{1}, f\left(e, x_{2}, f\left(e, y_{3}, e\right)\right)\right) \\
& =f\left(e, x_{1}, f\left(f\left(e, x_{2}, e\right), y_{3}, e\right)\right)=f\left(e, x_{1}, f\left(f\left(e, y_{2}, e\right), y_{3}, e\right)\right) \\
& =f\left(e, x_{1}, f\left(f\left(e, e, y_{2}\right), y_{3}, e\right)\right)=f\left(e, x_{1}, f\left(e, f\left(e, y_{2}, y_{3}\right), e\right)\right) \\
& \left.=f\left(f\left(e, x_{1}, e\right), f\left(e, y_{2}, y_{3}\right), e\right)\right)=f\left(f\left(e, y_{1}, e\right), f\left(e, y_{2}, y_{3}\right), e\right) \\
& =f\left(e, y_{1}, f\left(e, f\left(e, y_{2}, y_{3}\right), e\right)\right)=f\left(e, y_{1}, f\left(f\left(e, e, y_{2}\right), y_{3}, e\right)\right) \\
& =f\left(f\left(e, y_{1}, f\left(e, e, y_{2}\right)\right), y_{3}, e\right)=f\left(f\left(e, f\left(y_{1}, e, e\right), y_{2}\right), y_{3}, e\right) \\
& =f\left(f\left(e, y_{1}, y_{2}\right), y_{3}, e\right)=f\left(e, f\left(y_{1}, y_{2}, y_{3}\right), e\right) .
\end{aligned}
$$

Thus, there exists $y \in f\left(y_{1}, y_{2}, y_{3}\right)$ such that $x \in f(e, y, e)=f(e, e, y)$. Consequently,

$$
f(e, x, e) \subseteq f(e, f(e, e, y), e)=f(f(e, e, e), y, e)=f(e, y, e) .
$$

Since $P_{e_{2}}$ is a partition of $H$, we have $f(e, x, e)=f(e, y, e)$. Hence, $x R y$ and the desired result follows.

(2) By Theorem 3.1 of [7], $H / R$ is a ternary hypergroup. Let $x \in H$ be an arbitrary element. We claim that $R(x)=f(e, x, e)$ which will imply that $R(e)=\{e\}$. For each $y \in R(x)$, we have $y \in f(e, y, e)=f(e, x, e)$. So, $R(x) \subseteq f(e, x, e)$. Conversely, for each $y \in f(e, x, e)$ we have $f(e, y, e) \subseteq f(e, f(e, x, e), e)=f(e, e, x)=f(e, x, e)$. Since $P_{e_{2}}$ is a partition of $H$, we have $f(e, y, e)=f(e, x, e)$ that is $y \in R(x)$. So, $f(e, x, e) \subseteq R(x)$. Therefore, $R(x)=f(e, x, e)$.

By using Lemma 3.4, we have $x \in f(x, e, e) \cap f(e, x, e) \cap f(e, e, x)$, for all $x \in H$. Thus, for each $R(x) \in H / R$, we have

$$
\left.\left.\left.R(x) \in f\right|_{R}(R(x), R(e), R(e)) \cap f\right|_{R}(R(e), R(x), R(e)) \cap f\right|_{R}(R(e), R(e), R(x)) .
$$

Thus, $R(e)$ is an identity element of $\left(H / R,\left.f\right|_{R}\right)$.

Let $R(x)$ be an arbitrary element of $H / R$. By Theorem 4.1, $(H, f)$ is right reversible. Thus, there exists $y \in H$ such that $e \in f(x, e, y) \cap f(y, e, x) \cap f(x, y, e)$. This implies that

$$
\left.\left.\left.R(e) \in f\right|_{R}(R(x), R(e), R(y)) \cap f\right|_{R}(R(y), R(e), R(x)) \cap f\right|_{R}(R(x), R(y), R(e)) .
$$

Hence, $R(y) \in(R(x))^{-1}$. So, $\left(H / R,\left.f\right|_{R}\right)$ is a regular ternary hypergroup.

Finally, let $R(x), R(y), R(z) \in H / R$ and $\left.R(x) \in f\right|_{R}(R(y), R(e), R(z))$. Then, there exists $t \in f(y, e, z)$ such that $R(x)=R(t)$. Since $(H, f)$ is right reversible, there exists $w \in z^{-1}$ such that $y \in f(t, e, w)$. So,

$$
\left.R(y) \in f\right|_{R}(R(t), R(e), R(w))=\left.f\right|_{R}(R(x), R(e), R(w)) .
$$


Clearly, $R(w) \in(R(z))^{-1}$. This proves that $H / R$ is right reversible.

(3) First, we prove (a).

(i) $\Rightarrow$ (ii) Let $\left.R(x) \in f\right|_{R}(R(y), R(z), R(t))$. As $R(y)=R(u), R(z)=R(v)$ and $R(t)=R(w)$, we have $\left.R(x) \in f\right|_{R}(R(u), R(v), R(w))$. Thus, there exists $a \in f(u, v, w)$ such that $R(x)=R(a)$. So, $a \in R(x) \cap f(u, v, w)$.

(ii) $\Rightarrow$ (iii) Let $a \in R(x) \cap f(u, v, w)$. Then,

$$
R(x)=R(a)=f(e, a, e) \subseteq f(e, f(u, v, w), e) .
$$

(iii) $\Rightarrow$ (i) According to hypothesis, we have $x \in f(e, f(u, v, w), e)$. Thus, there exists $a \in f(u, v, w)$ such that $x \in f(e, a, e)$. Now, from $R(a)=f(e, a, e)$ it follows that $R(x)=R(a)$. On the other hand, $\left.R(a) \in f\right|_{R}(R(u), R(v), R(w))$ which implies that $\left.R(x) \in f\right|_{R}(R(y), R(z), R(t))$.

Proof of (b) is trivial.

In what follows, when $(H, f)$ is a finite ternary hypergroup of type $\mathrm{U}$ on the right, we denote by $m_{e_{1}}$ and $m_{e_{2}}$ the maximum size of the elements of $P_{e_{1}}$ and $P_{e_{2}}$, respectively.

Proposition 4.1. Let $(H, f, e)$ be a finite ternary hypergroup of type $U$ on the right. Then, the following assertions hold.

(1) $m_{e_{1}}=1$ if and only if $e$ is a left scalar identity.

(2) If $m_{e_{1}} \geq 2$, then there exist two distinct elements $x, y \in H \backslash\{e\}$ such that $f(e, e, x)=f(e, e, y)$ and $|f(e, e, x)|=|f(e, e, y)|=m_{e_{1}}$.

(3) If $m_{e_{2}} \geq 2$ and $e$ is a central identity, then there exist two distinct elements $x, y \in H \backslash\{e\}$ such that $f(e, x, e)=f(e, y, e)$ and $|f(e, x, e)|=\mid f\left(e, y, e \mid=m_{e_{2}}\right.$.

Proof. (1) By using Lemma 3.2, the proof is trivial.

(2) If $m_{e_{1}} \geq 2$, then there exists $x \in H \backslash\{e\}$ such that $|f(e, e, x)|=m_{e_{1}}$. Thus, by Lemma 3.3, there exists $y \in H \backslash\{x\}$ such that $x \in f(e, e, y)$. Consequently, $f(e, e, x) \subseteq f(e, e, y)$ and $m_{e_{1}}=|f(e, e, x)| \leq|f(e, e, y)|$. Since $m_{e_{1}}$ is maximal, we obtain $|f(e, e, x)|=|f(e, e, y)|$ and therefore $f(e, e, x)=f(e, e, y)$.

(3) If $m_{e_{2}} \geq 2$, then there exists $x \in H \backslash\{e\}$ such that $|f(e, x, e)|=m_{e_{2}}$. Thus, by Lemma 3.5, there exists $y \in H \backslash\{x\}$ such that $x \in f(e, y, e)$. Since $e$ is a central identity, from Lemma 3.4 it follows that $f(e, e, y)=f(e, y, e)$. Consequently, $f(e, x, e) \subseteq f(e, e, y)=f(e, y, e)$. So, $m_{e_{2}}=|f(e, x, e)| \leq|f(e, y, e)|$. Since $m_{e_{2}}$ is maximal, we obtain $|f(e, x, e)|=|f(e, y, e)|$ and therefore $f(e, x, e)=f(e, y, e)$.

\section{REFERENCES}

[1] S. M. Anvariyeh, S. Mirvakili and B. Davvaz, Combinatorial aspects of $n$-ary polygroups and $n$-ary color schemes, European J. Combin. 34 (2013), 207-216.

[2] S. M. Anvariyeh, B. Davvaz and S. Mirvakili, Construction of n-ary (H, G)-hypergroups, Port. Math. 69(4) (2012), 259-281.

[3] P. Corsini and V. Leoreanu, Applications of Hyperstructures Theory, Advanced in Mathematics, Kluwer Academic Publisher, 2003.

[4] B. Davvaz, Polygroup Theory and Related Systems, World Scientific, 2013. 
[5] B. Davvaz and V. Leoreanu-Fotea, Hyperring Theory and Applications, International Academic Press, USA, 2007.

[6] B. Davvaz and V. Leoreanu-Fotea, Binary relations on ternary semihypergroups, Comm. Algebra 38 (2010), 3621-3636.

[7] B. Davvaz and T. Vougiouklis, n-ary hypergroups, Iran. J. Sci. Technol. Trans. A Aci. 30(2) (2006), 165-174.

[8] B. Davvaz, W. A. Dudek and S. Mirvakili, Neutral elements, fundamental relations and n-ary hypersemigroups, Internat. J. Algebra Comput. 19(4) (2009), 567-583.

[9] W. A. Dudek, B. Davvaz and T. Vougiouklis, A generalization of n-ary algebraic systems, Comm. Algebra 37 (2009), 1248-1263.

[10] D. Fasino and D. Freni, Minimal order semihypergroups of type $U$ on the right, Mediterr. J. Math. 5 (2008), 295-314.

[11] D. Freni, Minimal order semihypergroups of type $U$ on the right, II, J. Algebra 340 (2011), 77-89.

[12] D. Freni, Structure des hypergroupes quotients et des hypergroupes de type U, Ann. Sci. Univ. Clermont-Ferrand II Math. 22 (1984), 51-77.

[13] D. Freni, Hypergroups of type $U$ and subhypergroups generated by a subset, Riv. Mat. Univ. Parma 13(4) (1987), 29-41.

[14] D. Freni, A note on the right scalar identity of a hypergroup of type $U$ on the right, Matematiche (Catania) 47 (1992), 75-81.

[15] D. Freni, M. de Salvo and G. Lo Faro, A new family of hypergroups and hypergroups of type $U$ on the right of size five, Far East J. Math. Sci. (FJMS) 26 (2007), 393-418.

[16] D. Freni, M. De Salvo and G. Lo Faro, Hypergroups of type U on the right of size five. II, Mat. Vesnik 60 (2008), 23-45.

[17] V. Leoreanu-Fotea and B. Davvaz, Join n-spaces and lattices, J. Mult.-Valued Logic Soft Comput. 15 (2009), 421-432.

[18] V. Leoreanu-Fotea and B. Davvaz, n-hypergroups and binary relations, European J. Combin. 29 (2008), 1207-1218.

[19] V. Leoreanu-Fotea and B. Davvaz, Roughness in n-ary hypergroups, Inform. Sci. 178 (2008), 4114-4124.

[20] F. Marty, Sur une generalization de la notion de group, In 8th Congress Math. Scandenaves, (1934), 45-49.

[21] S. Mirvakili and B. Davvaz, Application of fundamental relations on n-ary Polygroups, Bull. Iranian Math. Soc. 38 (2012), 169-184.

[22] S. Mirvakili and B. Davvaz, Applications of strongly transitive geometric spaces to n-ary hypergroups, ARS Combin. 109 (2013), 193-227.

[23] T. Vougiouklis, Hyperstructures and Their Representations, Hadronic Press Inc., Palm Harbor, USA, 1994.

[24] B. N. Waphare and M. Ghadiri, n-ary polygroups, Iran. J. Sci. Technol. Trans. A Sci. 33 (2009), $145-158$.

\author{
${ }^{1}$ Department Mathematics \\ YAZD UNIVERSITY \\ YAZD, IRAN \\ E-mail address: davvaz@yazd.ac.ir
}

